\title{
BMJ Open Effects of positive psychology interventions in Arab countries: a protocol for a systematic review
}

\author{
Asma Basurrah (D) , ${ }^{1,2}$ Louise Lambert, ${ }^{3}$ Annalisa Setti, ${ }^{1}$ Mike Murphy, ${ }^{1}$ \\ Meg Warren, ${ }^{4}$ Topaz Shrestha, ${ }^{1}$ Zelda di Blasi ${ }^{1}$
}

To cite: Basurrah A, Lambert L, Setti A, et al. Effects of positive psychology interventions in Arab countries: a protocol for a systematic review. BMJ Open 2021;11:e052477. doi:10.1136/ bmjopen-2021-052477

- Prepublication history and additional supplemental material for this paper are available online. To view these files, please visit the journal online (http://dx.doi.org/10.1136/ bmjopen-2021-052477)

Received 16 April 2021 Accepted 14 July 2021

Check for updates

(C) Author(s) (or their employer(s)) 2021. Re-use permitted under CC BY-NC. No commercial re-use. See rights and permissions. Published by BMJ.

${ }^{1}$ School of Applied Psychology, University College Cork, Cork, Ireland

${ }^{2}$ Faculty of Arts and Humanities, Department of Psychology, King Abdulaziz University, Jeddah, Saudi Arabia

${ }^{3}$ Psychology Department, Canadian University of Dubai, Dubai, UAE

${ }^{4}$ College of Business and Economics, Department of Management, Western Washington University, Bellingham, Washington, USA

Correspondence to

Asma Basurrah;

116106685@umail.ucc.ie

\section{ABSTRACT}

Introduction Despite the growing volume of published studies on the effects of positive psychology interventions (PPIs), little is known about their effectiveness outside of Western countries, particularly in Arab countries. As the effectiveness of PPIs in this region remains unclear, a systematic review focusing on this area of research can offer a valuable contribution. Here, we present a protocol for the first systematic review that aims to examine the effects of PPIs on increasing well-being, quality of life and resilience and decreasing depression, anxiety and stress for both health and clinical, child and adult populations in Arab countries.

Methods and analysis This protocol is carried out in accordance with the Preferred Reporting Items for Systematic Reviews and Meta-Analyses Protocols guidelines. A systematic literature search for studies up to 30 April 2021 will be conducted in the following electronic databases: PsycINF0, PubMed, Scopus, ProQuest, Dar Al Mandumah and AI Manhal. Experimental/ quasi-experimental quantitative studies evaluating the effects of PPIs on healthy and clinical participants of all ages in the 22 Arab countries will be included. Outcomes will include psychological effects of PPIs on dimensions related to well-being (eg, happiness), quality of life, resilience, depression, anxiety and stress. The risk of bias will be evaluated using the Cochrane risk-of-bias tool. A narrative synthesis with tables of study characteristics will be provided. A meta-analysis will be included if outcomes allow; in this instance, subgroups analysis will be conducted, depending on the data gathered, to examine differences in effect sizes based on age group, population type, duration of intervention and type of intervention. Ethics and dissemination Ethical approval was not required for the performance of this systematic review. We intend to publish the study in a peer-reviewed journal and share the findings at relevant conferences.

PROSPERO registration number CRD42020198092.

\section{INTRODUCTION}

The Arab region accounts for around 5\% of the world's population. As of 2019, this region was home to nearly 427 million inhabitants, ${ }^{1}$ with $60 \%$ being aged under 25 years. Arab countries have recorded the highest burden of mental health disorders globally. ${ }^{2}$ In a call for action into mental health

\section{Strengths and limitations of this study}

This will be the first systematic review to provide an evidence-based review of the effects of positive psychology interventions for clinical and healthy populations in the Arab region.

- The main strength is the inclusion, in addition to the English databases, of the two largest Arabic databases.

- An extensive search strategy was developed in consultation with a review team as well as a library specialist for both searches concerning English and translated Arabic terms.

- A description of the intervention types, durations, delivery methods and population types will be provided, enabling investigation of their effectiveness.

research in the Arab region published in the Lancet, researchers explained that stigma, reluctance to self-disclose and to seek formal help, conflict and war were some of the reasons for such high levels of mental health disorders. ${ }^{3}$

With the increasing population growth in the region, research directly studying positive mental health and well-being is needed. This research will in turn impact the world, especially with the increasing concern regarding mental health problems caused by the COVID-19 pandemic. ${ }^{4}$ Several studies have examined the psychological impact of the ongoing pandemic and reported negative effects on mental health including anxiety, depression and stress. ${ }^{5-8}$ These findings emphasise the need to support people during this time through delivering psychological interventions. ${ }^{4-7}$ As positive psychology interventions (PPIs) focus on cultivating psychological resilience and well-being as well as alleviating mental health problems, ${ }^{9-14}$ we believe the findings of this review are an important contribution in addressing mental health problems resulting from the COVID-19 pandemic. 
The past decade has witnessed a rise in research examining PPIs. ${ }^{15}$ Sin and Lyubomirsky first defined PPIs as intervention, therapy or activity primarily aimed at increasing positive feelings, positive behaviours or positive cognitions'. ${ }^{9}$ Bolier et al assert that PPIs should be designed based on positive psychology theories. ${ }^{10}$ Another definition was proposed by Parks and Biswas-Diener in which they emphasise that interventions must target 'positive' variables and have sufficient empirical evidence. ${ }^{16}$ PPIs include, but are not restricted to, gratitude, compassion, strengths, optimism and kindness. While a variety of definitions have been suggested, we define PPIs as psychological interventions (training and therapy) aimed at enhancing positive feelings, behaviours or cognitions, based on positive psychology theories and research.

Several meta-analyses have found that PPIs have a small to moderate significant effect on well-being and distress in both the general population (eg, 910 12) and patients with mental health problems (eg, 11). These include meta-analyses that have examined single PPIs (eg, 9 10) and multicomponent $($ eg, 12) PPIs. While studies on PPIs have been mainly examined in Western countries, dominated by Western, educated, industrialised, rich and democratic populations, ${ }^{17}$ there is now some evidence for their effectiveness in non-Western countries. ${ }^{18}$ In a separate systematic review of PPIs in nonWestern countries, Hendriks et al reported a moderate effect for well-being and a large effect for depression and anxiety. ${ }^{18}$ This review was limited to peer-reviewed English articles published up until 2017. In 2020, Carr et al conducted a meta-analysis including studies published in any language in peer-reviewed journals or grey literature. ${ }^{19}$ On review of 347 studies, including three studies from the Arab region, they reported a small to medium significant effect of PPIs on wellbeing and distress. ${ }^{19}$ They concluded that those who benefitted most from multiple PPIs were clinical samples from non-Western countries, who engaged in the programmes for longer periods. However, they did not search Arabic databases (eg, Dar Al Mandumah), where most Arabic studies can be found. The present review is designed to address this limitation.

The Arab world consists of 22 countries in the Middle East and North Africa: Algeria, Bahrain, Comoros, Djibouti, Egypt, Iraq, Jordan, Kuwait, Lebanon, Libya, Morocco, Mauritania, Oman, Palestine, Qatar, Saudi Arabia, Somalia, Sudan, Syria, Tunisia, the United Arab Emirates and Yemen. These countries share cultural traditions, histories and a common language. Researchers from the Middle East and North Africa have recently shown an increasing interest in positive psychology. A systematic review investigating the prevalence and characteristics of positive psychology research in the Middle East and North Africa region, published in 2015, was undertaken by Rao, Donaldson and Doiron. ${ }^{20}$ On reviewing a total of 53 studies, they found that positive psychology research in the region has grown exponentially since 2011 . They also found that the region's literature focused on two paths: one path aimed at increasing positive states, while the other path aimed to coping with adversity. However, this review by Rao and colleagues did not examine the effects of PPIs. ${ }^{20}$ The current review attempts to address this gap.

The positive psychology movement originated in the USA, ${ }^{21}$ which raises concerns about the practical generalisability of PPIs, due to cultural differences between Western 'individualistic' and Eastern 'collectivist' cultures. ${ }^{22}{ }^{23}$ Because of these differences, we believe it is important to undertake this review. Several studies across the Arab region have recently examined the effects of PPIs, ${ }^{24-29}$ with studies adapting these interventions to their particular cultures. ${ }^{25} 3031$ However, the effects of PPIs in the Arab region remain unclear as, to our knowledge, no systematic quantitative review has yet been published. It is timely and essential to provide the Arab populations with evidence on PPIs to develop a culturally responsive positive psychology, ${ }^{32}$ as well as an indigenous positive psychology. ${ }^{33}$

\section{Objectives}

Primary objective

- To examine the effectiveness of PPIs on increasing well-being, quality of life and resilience and decreasing depression, anxiety and stress for both health and clinical, child, adolescent and adult populations in the Arab region.

\section{Secondary objectives}

- To identify types of PPIs that have been conducted in the Arab region.

- To determine if the interventions were adapted for the local context.

- To identify variables that may influence the effects of PPIs on outcomes.

\section{METHODS AND ANALYSIS}

This protocol was developed in accordance with the Preferred Reporting Items for Systematic Reviews and Meta-Analyses Protocols (PRISMA-P) guidelines. ${ }^{34}$ This review has been registered in the International Prospective Register of Systematic Reviews-PROSPERO. In the event of protocol amendments, the date of each amendment will be recorded and reported in PROSPERO with a description of changes and its rationale.

Criteria for considering studies for this review.

\section{Types of studies}

We will include randomised controlled trials and quasiexperiments (controlled, non-randomised and pre/ postintervention studies).

Non-experimental studies (eg, uncontrolled, cohort, descriptive and observational) and qualitative studies will be excluded. We will include studies published in peerreviewed journals and dissertations.

\section{Types of participants}

\section{Inclusion}

Healthy and clinical participants of all ages in the Arab region. The Arab countries include Algeria, Bahrain, Comoros, Djibouti, Egypt, Iraq, Jordan, Kuwait, Lebanon, Libya, Morocco, Mauritania, Oman, Palestine, Qatar, 
Saudi Arabia, Somalia, Sudan, Syria, Tunisia, the United Arab Emirates and Yemen.

\section{Exclusion}

Participants from outside the Arab region. When a study includes Arab and non-Arab participants, the study is included if the results of the Arab participants are presented separately.

\section{Patient and public involvement}

No patient involved. Data will be collected from published articles.

\section{Types of interventions}

Inclusion: Studies will be eligible for inclusion if they investigated the delivery of intervention (training and therapy) aimed at enhancing positive feelings, positive behaviours or positive cognitions. We will include studies examining one intervention (single component) or two or more interventions (multicomponent). The intervention must be explicitly developed in line with the theoretical tradition of positive psychology. PPIs are defined by Sin and Lyubomirsky as 'treatment methods or intentional activities that aim to cultivate positive feelings, behaviours or cognitions' (p. 467) ${ }^{9}$ PPIs include, but are not restricted to, self-compassion, gratitude, character strengths, mindfulness, optimism (eg, best possible self), forgiveness, kindness, savouring and humour.

Exclusion: We will exclude studies reporting the effects of physical activity interventions. Studies examining traditional psychotherapeutic interventions (eg, cognitive behavioural therapy) including a component of positive psychology will also be excluded.

\section{Types of outcome measures}

The outcomes of interest are well-being (eg, happiness and life satisfaction), quality of life, resilience, depression, anxiety and stress. We will include only studies that reported changes in at least one of those psychological outcome measures, assessed preintervention and postintervention and linked their findings to positive psychology literature.

Search method for identification of studies.

\section{Electronic searches}

For English-language literature, the databases that will be searched are the following:

- PsycINFO.

- MEDLINE (via PubMed).

- Scopus.

- ProQuest Dissertations and theses.

For Arabic-language literature, the databases that will be searched are the following:

- Dar Al Mandumah.

- Al Manhal.

Limits will be applied to retrieve studies published in the English or Arabic language from 1998 - the inception of the positive psychology movement-to 30 April 2021. The searches will be rerun just before the final analyses, and new studies will be retrieved for inclusion. Manual searches of references will be conducted in relevant papers. We will also search PROSPERO and the Cochrane Library for any systematic reviews planned or completed. A range of words and indexed terms related to 'positive psychology interventions' and 'Arab countries' will be searched. The strategies for searching databases will be modelled on the search strategy designed for PubMed (see online supplemental file 1 , search strategy example). In order for us to develop equivalent search terms in Arabic, AB, the first author, who is a native Arabic speaker, will translate the English search terms in consultation with experts in the field.

\section{Searching other resources}

- Reference lists of recent meta-analyses ${ }^{12} 1819$ and a review from the Middle East and North Africa region ${ }^{20}$ will be searched.

- We will contact experts in the field from the Arab region and ask them to provide sources that might still be missing.

- Hand searching of the Middle East Journal of Positive Psychology, as well as of the recent book called Positive Psychology in the Middle East/North Africa ${ }^{35}$ will be carried out. Reference lists of all eligible studies will be hand-searched to attempt to identify additional relevant studies.

\section{Study records}

\section{Data management and selection process}

Search results, including citations, abstracts and full-text articles, will be uploaded and recorded to EndNote X9. We will remove duplicates and screen all titles and abstracts against the inclusion criteria. This will be done by $\mathrm{AB}$, and then, a second reviewer will screen a random $10 \%$ of studies independently to ensure consistency. The kappa statistic will be calculated to quantify the interobserver agreement. Where titles and abstracts are deemed to be relevant or unclear, fulltext articles will be retrieved and independently screened by two reviewers to identify studies for inclusion. Additional information will be obtained if required. In case of disagreement, a third reviewer will be involved. Studies that are noted as excluded will be recorded, and their reason for exclusion will be reported using a flow diagram following the PRISMA-P guidelines.

\section{Data extraction process}

A data extraction form will be developed and piloted to obtain outcome data from included studies. This will be done by $\mathrm{AB}$, and another reviewer will check the extracted data. Discrepancies will be resolved by discussion. In case of disagreement, the final classification will be made by consensus with the involvement of a third reviewer. Extracted data will be recorded in an excel spreadsheet. Data extracted will include the following:

1. Country of origin, author(s) and year of publication.

2. Study method: design (eg, experimental and quasi-experimental). 
3. Sample (eg, number of participants, clinical or nonclinical and gender).

4. Type of intervention: single component versus multicomponent.

5. Delivery form.

6. Session duration (number of sessions and duration of session period).

7. Control group.

8. Number of participants at follow-up.

9. Mean/SD, p value and effect size.

10. Retention rate (post).

11. Outcome measures, questionnaires used.

\section{Dealing with missing data}

In the case of missing data or insufficient information, we will attempt to contact the study authors. If the authors cannot be contacted, available data will be analysed as reported.

\section{Assessment of risk of bias in included studies}

We will use the Cochrane risk-of-bias tool, in accordance with the Cochrane Handbook, to assess the methodological quality of the included studies. This will be done by two reviewers independently. In the case of disagreements, a discussion will be conducted with a third reviewer to reach a consensus. In the case of insufficient or additional information, we will contact the study authors. The assessments will be classified into three levels: low risk, some concerns and high risk.

\section{Data synthesis}

We will provide a narrative synthesis of all the included studies' findings, with tables of study characteristics, participants, intervention details and outcome measures. Where possible, quantitative data will be pooled for a meta-analysis. Multilevel modelling will be conducted to synthesise multiple effect sizes from single studies.

\section{Subgroup analysis}

Depending on the data gathered, subgroups may be formed and outcomes explored according to age or clinical nature of samples. Subgroup analyses will be conducted to examine moderating effects of the following possible moderators. The moderators are as follows:

1. Age group: child/adolescent (up to 17 years old) or adult (18 years old and up).

2. Study population: clinical or non-clinical.

3. Type of intervention: single component or multicomponent.

4. Duration of intervention: short ( $<8$ weeks) or long ( $>8$ weeks).

\section{Ethics and dissemination}

This systematic review will use data from published literature; hence, no ethical approval will be required. The results of this systematic review will be submitted to a peer-reviewed international journal and shared at relevant conferences.

\section{DISCUSSION}

PPIs have the potential to improve mental health and promote well-being. However, much uncertainty still exists in examining the application of these interventions in the Arab region, as most studies have only focused on Western samples. This review will be the first study to systematically review the efficacy of PPIs in the Arab region. The findings of this review are expected to provide health/clinical populations of all ages in the Arab region with a detailed and evidence-based overview of the overall effects of PPIs that will enrich the field of positive psychology and mental health. In turn, this will contribute to evaluating these types of interventions and strengthen their generalisability by providing a multicultural perspective ${ }^{33}$ for health professionals and practitioners in the field.

Acknowledgements This systematic review is part of a $\mathrm{PhD}$ thesis. The first author, $A B$, is supported by the graduate scholarship programme of King Abdulaziz University, Ministry of Higher Education, Saudi Arabia. The authors thank the editor and reviewers of the manuscript for their constructive feedback and the support of Donna 0'Doibhlin who is a librarian and was instrumental in providing advice on structuring the keyword search.

Contributors $\mathrm{AB}$ and $\mathrm{ZdB}$ conceptualised, designed and registered the protocol and developed the search strategy with the assistance of an information specialist and contributions from coauthors (MM, AS, MW and LL). AB will screen potential studies, extract data, assess the risk of bias and complete data synthesis, along with a second reviewer (TS). Statistical analysis will be conducted by MM. All coauthors (AB, ZdB, MM, AS, MW, LL and TS) critically revised the protocol, provided feedback and approved the final manuscript.

Funding The authors have not declared a specific grant for this research from any funding agency in the public, commercial or not-for-profit sectors.

Competing interests None declared.

Patient consent for publication Not required.

Provenance and peer review Not commissioned; externally peer reviewed.

Supplemental material This content has been supplied by the author(s). It has not been vetted by BMJ Publishing Group Limited (BMJ) and may not have been peer-reviewed. Any opinions or recommendations discussed are solely those of the author(s) and are not endorsed by BMJ. BMJ disclaims all liability and responsibility arising from any reliance placed on the content. Where the content includes any translated material, BMJ does not warrant the accuracy and reliability of the translations (including but not limited to local regulations, clinical guidelines, terminology, drug names and drug dosages), and is not responsible for any error and/or omissions arising from translation and adaptation or otherwise.

Open access This is an open access article distributed in accordance with the Creative Commons Attribution Non Commercial (CC BY-NC 4.0) license, which permits others to distribute, remix, adapt, build upon this work non-commercially, and license their derivative works on different terms, provided the original work is properly cited, appropriate credit is given, any changes made indicated, and the use is non-commercial. See: http://creativecommons.org/licenses/by-nc/4.0/.

ORCID iD

Asma Basurrah http://orcid.org/0000-0002-4495-1612

\section{REFERENCES}

1 World Bank, World Development Indicators. DataBank (population total), 2020. Available: https://databank.worldbank.org/reports.aspx? source $=2 \&$ series $=$ SP.POP.TOTL\&country

2 GBD 2015 Eastern Mediterranean Region Mental Health Collaborators. The burden of mental disorders in the Eastern Mediterranean region, 1990-2015: findings from the global burden of disease 2015 study. Int J Public Health 2018;63:25-37.

3 Maalouf FT, Alamiri B, Atweh S, et al. Mental health research in the Arab region: challenges and call for action. Lancet Psychiatry 2019;6:961-6. 
4 Shuwiekh HAM, Kira IA, Sous MSF, et al. The differential mental health impact of COVID-19 in Arab countries. Curr Psychol 2020;11:1-5.

5 Rajkumar RP. COVID-19 and mental health: a review of the existing literature. Asian J Psychiatr 2020;52:102066.

6 Alkhamees AA, Alrashed SA, Alzunaydi AA, et al. The psychological impact of COVID-19 pandemic on the general population of Saudi Arabia. Compr Psychiatry 2020;102:152192.

7 Jia R, Ayling K, Chalder T, et al. Mental health in the UK during the COVID-19 pandemic: cross-sectional analyses from a community cohort study. BMJ Open 2020;10:e040620

8 Xiong J, Lipsitz O, Nasri F, et al. Impact of COVID-19 pandemic on mental health in the general population: a systematic review. J Affect Disord 2020;277:55-64.

9 Sin NL, Lyubomirsky S. Enhancing well-being and alleviating depressive symptoms with positive psychology interventions: a practice-friendly meta-analysis. J Clin Psychol 2009;65:467-87.

10 Bolier L, Haverman M, Westerhof GJ, et al. Positive psychology interventions: a meta-analysis of randomized controlled studies. BMC Public Health 2013;13:119.

11 Chakhssi F, Kraiss JT, Sommers-Spijkerman M, et al. The effect of positive psychology interventions on well-being and distress in clinical samples with psychiatric or somatic disorders: a systematic review and meta-analysis. BMC Psychiatry 2018;18:1-17.

12 Hendriks T, Schotanus-Dijkstra M, Hassankhan A, et al. The efficacy of multi-component positive psychology interventions: a systematic review and meta-analysis of randomized controlled trials. J Happiness Stud 2020;21:357-90.

13 Rashid T, McGrath RE. Strengths-based actions to enhance wellbeing in the time of COVID-19. Int J Wellbeing 2020;10:113-32.

14 Matiz A, Fabbro F, Paschetto A, et al. Positive impact of mindfulness meditation on mental health of female teachers during the COVID-19 outbreak in Italy. Int J Environ Res Public Health 2020;17:6450.

15 Rusk RD, Waters LE. Tracing the size, reach, impact, and breadth of positive psychology. J Posit Psychol 2013;8:207-21.

16 Parks AC, Biswas-Diener R. Positive interventions: past, present and future. In: Mindfulness, acceptance, and positive psychology: the seven foundations of well-being, 2013: 140-65.

17 Hendriks T, Warren MA, Schotanus-Dijkstra M, et al. How WEIRD are positive psychology interventions? A bibliometric analysis of randomized controlled trials on the science of well-being. $J$ Posit Psychol 2019;14:489-501.

18 Hendriks T, Schotanus-Dijkstra M, Hassankhan A, et al. The efficacy of positive psychology interventions from non-western countries: a systematic review and metaanalysis. Int J Wellbeing 2018;8:71-98.

19 Carr A, Cullen K, Keeney C. Effectiveness of positive psychology interventions: a systematic review and meta-analysis. J Posit Psychol 2020;12:1-21.

20 Rao MA, Donaldson SI, Doiron KM. Positive psychology research in the Middle East and North Africa. Middle East J Posit Psychol 2015;1:60-76.
21 Seligman ME, Csikszentmihalyi M. Positive psychology: an introduction. In: Flow and the foundations of positive psychology. Springer: Dordrecht, 2014

$22 \mathrm{Ng} \mathrm{W}$, Lim WS. Developing positive psychological interventions: maximizing efficacy for use in eastern cultures. In: Positive psychological intervention design and protocols for multi-cultural contexts. Cham: Springer, 2019.

23 Wong PT. Positive psychology. Encyclopedia Cross-Cultural Psychol 2013:3:1021-7.

24 Barrington N, Hancock R, Clough P. Impact of a resilience programme on pupil anxiety, depression and mental Toughness. Middle East J Posit Psychol 2019;5:60-81.

25 Al-Ghalib S, Salim A. A mindfulness based intervention to enhance university student wellbeing in Saudi Arabia. Middle East J Posit Psychol 2018;4:142-57

26 Lambert L, Passmore H-A, Joshanloo M. A positive psychology intervention program in a culturally-diverse university: boosting happiness and reducing fear. J Happiness Stud 2019;20:1141-62.

27 Basurrah AA, O'Sullivan D, Chan JS. A character strengths intervention for happiness and depression in Saudi Arabia: A replication of Seligman, et al.'s (2005) study. Middle East J Posit Psychol 2020;6:41-72.

28 Ramadan HA. The effectiveness of a therapeutic program to promote subjective wellbeing by using multi-components of positive psychology interventions. J Arabic Stud Educat Psychol 2014;54:251-80.

29 Al-Wakeel S. Efficacy of using some techniques of positive psychology in improving level of the psychological adjustment for a sample of diabetic patients. J Educat 2010;2:118-56.

30 Lambert L, Passmore H-A, Scull N, et al. Wellbeing matters in Kuwait: the Alnowair's Bareec education initiative. Soc Indic Res 2019;143:741-63.

31 Rayan A, Ahmad M. Effectiveness of mindfulness-based interventions on quality of life and positive reappraisal coping among parents of children with autism spectrum disorder. Res Dev Disabil 2016;55:185-96.

$32 \mathrm{Kim} \mathrm{H}$, Doiron K, Warren MA, et al. The international landscape of positive psychology research: a systematic review. Int $J$ Wellbeing 2018;8:50-70.

33 Pandey S. Positive psychology: blending strengths of western, eastern and other indigenous psychologies. In: 1st Internationa Conference on "Emerging Paradigms in Business \& Socia Sciences"(EPBSS-2011), organized by Middlesex University, Dubai 2011.

34 Moher D, Shamseer L, Clarke M, et al. Preferred reporting items for systematic review and meta-analysis protocols (PRISMA-P) 2015 statement. Syst Rev 2015;4:1-9.

35 Lambert L, Pasha-Zaidi N. Positive psychology in the Middle East/ North Africa. Springer International Publishing, 2019. 